\title{
A Game-Theoretic Framework for Interference Management Through Cognitive Sensing
}

\author{
Yingda Chen, Koon Hoo Teo, Shalinee Kishore, Jinyun Zhang
}

TR2008-060 September 2008

\begin{abstract}
A game theoretic framework is developed in this paper to facilitate inter-cell interference management through cognitive sensing distributively performed by mobile stations (MSs). Using stochastic geometry, we reveal the relationship between the effectiveness of interference management and MSś ẅillingnessto perform cognitive sensing. Such cognitive sensing performed by MS is motivated by the associated beneficial results as well as by the rewards from base stations (BS) that encourage sensing. Different tradeoffs for BS and MSs exist in their interactions, which are modeled as a stackelberg game in this paper. While both BS and MS seek to manage interference at its own minimum cost, we design algorithm to achieve nash Equilibrium in such a game and investigate the optimal strategies taken by the players (BS and MSs).
\end{abstract}

ICC 2008

This work may not be copied or reproduced in whole or in part for any commercial purpose. Permission to copy in whole or in part without payment of fee is granted for nonprofit educational and research purposes provided that all such whole or partial copies include the following: a notice that such copying is by permission of Mitsubishi Electric Research Laboratories, Inc.; an acknowledgment of the authors and individual contributions to the work; and all applicable portions of the copyright notice. Copying, reproduction, or republishing for any other purpose shall require a license with payment of fee to Mitsubishi Electric Research Laboratories, Inc. All rights reserved. 



\title{
A Game-theoretic Framework for Interference Management through Cognitive Sensing
}

\author{
Yingda Chen ${ }^{\dagger}$, Koon Hoo $\mathrm{Teo}^{\ddagger}$, Shalinee Kishore ${ }^{\dagger}$ and Jinyun Zhang ${ }^{\ddagger}$ \\ ${ }^{\dagger}$ Electrical and Computer Engineering, Lehigh University, Bethlehem, PA 18015 \\ ${ }^{\ddagger}$ Mitsubishi Electric Research Laboratories, Cambridge, MA 02139 \\ $\dagger\{$ Yingda, Skishore $\} @$ Lehigh.edu, ${ }^{\ddagger}\{$ Teo, Jzhang $\} @$ MERL.com
}

\begin{abstract}
A game theoretic framework is developed in this paper to facilitate inter-cell interference management through cognitive sensing distributively performed by mobile stations (MSs). Using stochastic geometry, we reveal the relationship between the effectiveness of interference management and MS's "willingness" to perform cognitive sensing. Such cognitive sensing performed by MS is motivated by the associated beneficial results as well as by the rewards from base station(BS) that encourage sensing. Different tradeoffs for BS and MSs exist in their interactions, which are modeled as a Stackelberg game in this paper. While both BS and MS seek to manage interference at its own minimum cost, we design algorithm to achieve Nash Equilibrium in such a game and investigate the optimal strategies taken by the players (BS and MSs).
\end{abstract}

\section{INTRODUCTION}

The "shortage" in available spectrum resource has accompanied the expanding need for the broadband wireless access in the past decade. Spectral efficient transmission schemes are therefore vital in designing future communication systems. In this regard, the Orthogonal Frequency Division Multiple Access (OFDMA) has gained widespread popularity as a promising technology in physical layer design for wireless systems, due to its high spectrum efficiency. It has been adopted in several future broadband systems such as WiMAX [1][2] and 3GPP-LTE [3][4]. For systems built upon OFDMA, intracell interference can be minimized as long as orthogonality among subcarriers is maintained. The management of intercell co-channel interference (i.e., collision of same subcarriers used in different cells), however, is yet to be properly addressed, especially when full spectrum reuse is implemented, the suppression of inter-cell interference is becoming more important in system design, to provide reliable services to the users, especially those located at cell boundary.

Most schemes recently developed to reduce inter-cell interference in OFDMA systems (such as WiMAX and 3GPP-LTE) stem from the channel assignments in conventional cellular systems [10], and are usually base-station-centric [5] [8]. These schemes typically require careful spectrum planning among different cells. For example, in soft frequency reuse [5], the spectrum is divided into multiple chunks, which are then allocated to mobile stations (MSs) located at the center and edge of the cell, respectively. The base stations (BSs) of adjacent cells coordinate beforehand to ensure that the "edge spectrum chunks" of adjacent cells differ. In partial frequency reuse [6][7], frequency reuse factor is set to be 1 for cell center MSs and 3 for cell edge MSs, which inevitably necessitates strict frequency planning. Those spectrum planning based approaches aforementioned usually requires a super centralized control above different BSs, which is even more difficult to implement when the BSs belong to different wireless operators and are independent of each other.

In this paper, we develop a MS-centric scheme for intercell interference management, through the means of cognitive sensing [12] performed by MSs. The scheme is built upon OFDMA downlink, but the methodologies developed herein apply to general multi-cell systems as well. In this scheme, distributive MSs sense their surrounding wireless environments and collect subcarrier usage information from the potential interferers (e.g., MSs in adjacent cells). When the subcarrier usage is quasi-static, the collected information will be valid for a period of time into the future, and can be compiled to reduce potential future interferences. While the task of cognitive sensing itself can be power-consuming for the MSs, the benefits are two-folded: Firstly, BS can provide certain amount of "reward" to encourage cognitive sensing; secondly, the information collected from cognitive sensing can eventually reduce interference to the MSs themselves. From the perspective of the BS, tradeoffs exist as well: while information collected from cognitive sensing will reduce power wasted in subcarrier collision, BS should decide whether such benefit justified the additional "rewards" given out. The interactions between BS and MSs are modeled in a game theoretic framework, particularly, as a Stackelberg game [11]. Both BS and MS are assumed to be independent entities in this game, in regard of decision making that involves cognitive sensing. Specifically, BS cannot enforce the task of cognitive sensing onto the battery-operated MSs. Instead, both parties decide on their optimal strategy by "rational thinking". The impact of the "extent" of cognitive sensing is first discussed using stochastic geometry [13], especially how it could affect the effectiveness of the interference management. The tradeoffs for BS and MS in the game are identified and used for proper modeling of the utility functions. The Nash Equilibrium of such game is derived and we also analyze the algorithm to achieve the Nash Equilibrium.

Immediately following this introduction, we describe the cognitive sensing model in Section II. The formulation of the game theoretic framework using Stackelberg game is discussed and analyzed in Section III and we conclude this paper in Section IV.

\section{SYSTEM DESCRIPTION}

We consider in this paper an OFDMA-based two-cell system with full spectrum reuse. The spectrum is divided into $S$ orthogonal subcarriers that form the set $\mathcal{S}$. Traffic load in cell $i$ 
is $\beta_{i} \in[0,1]$. The MSs in both cells are uniformly distributed according to a two-dimensional Possion point process [13], MSs within a cell are assumed to have the same statistical average on the number of subcarriers usage. Therefore, the distribution of subcarrier usage in a cell is also geographically uniform. Each of the two BSs covers a circular cell with radius $R$ and the two cells overlap $\alpha R$ in radius. We assume that interference occurs only in the overlapping area to which we refer it as the interference zone (IZ). The MSs from cell $i$ that are located in the IZ form the set $\mathcal{I}_{i}$ and the MSs in the IZ from cell $j$ form the set $\mathcal{I}_{j}$. For simplicity, we further assume that the MSs in $\mathcal{I}_{i}$ are only subject to interference from downlink transmission to MSs in $\mathcal{I}_{j}$, vice versa ${ }^{1}$.

When BSs coordinate with each other, interference management can be collectively carried out by both BSs. Examples of such schemes can be found in [5] [9]. Especially, a game theoretic study is provided in [9] to model interactions between adjacent BSs in interference management based on partial information exchange. In this paper, we assume that adjacent BSs are independent and therefore do not coordinate with each other. In addition, MSs registered with one BS cannot directly inform its subcarrier usage to another BS. We further assume that only entities in cell $i$ (including BS $i$ and MSs in $\mathcal{I}_{i}$ ) engage in unilateral efforts to suppress co-channel subcarrier collisions. In this case, BS $i$ can gather information on interferers (i.e., downlink transmissions to MSs in $\mathcal{I}_{j}$ ) through the cognitive sensing performed distributively by MSs in $\mathcal{I}_{i}$. Such cognitive sensing begins with a probing/sensing signal sent out from a MS $\ell \in \mathcal{I}_{i}$, which covers a circular sensing region $A_{\ell}$ of radius $r_{\ell}$. If a MS in $\mathcal{I}_{j}$ receives this probing signal, it will respond ${ }^{2}$ and report its presence to $\ell$ as well as the subcarriers it is currently using, if any.

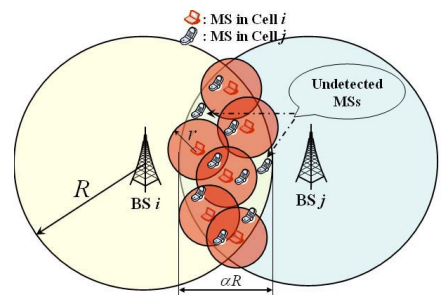

Fig. 1: Cognitive sensing in two-cell model

The distributive cognitive sensing by MSs in $\mathcal{I}_{i}$ is illustrated by Fig. 1. Assume there are $N_{i}$ MSs in cell $i$ (For the sake of clarity, only MSs in the IZ are shown in Fig. 1) and MSs in both cells are uniformly distributed. The distribution of MSs in cell $i$ can then be described by a two-dimensional Poisson

\footnotetext{
${ }^{1}$ Note that such assumption and simplification is taken here only for analytical and notational convenience. In general, when the MSs are not uniformly distributed, or when some MSs outside the overlapping area are subject to co-channel interference as well, the methodologies proposed herein still apply. The only difference lies in the definition of the interference zone, the set of $\mathcal{I}_{i}$ will differ as well and may lack an readily intuitive interpretation.

${ }^{2}$ In practice, it is not compulsory for a MS $\jmath \in \mathcal{I}_{j}$ to respond upon receiving the probing signal from $\ell$. However, since subcarrier collision is mutual and is equally detrimental to both MSs involved, $\jmath$ has the incentive to respond to probing request from $\ell$, since the response will eventually contribute to avoiding co-channel subcarrier collision (and is therefore beneficial to $J$ as well). In this paper, we focus on the interaction between BS $i$ and MSs in $\mathcal{I}_{i}$ and assume spontaneous response from $\jmath \in \mathcal{I}_{j}$. The interactions between MSs $\ell$ and $J$ can be modeled as a game-theoretic problem in its own right, but does not fall within the scope of discussions in this paper.
}

point process with density $\lambda_{i}=\frac{N_{i}}{\pi R^{2}}$. In such Poisson point process, if we let $N_{A}$ be the number of MSs from $\mathcal{I}_{i}$ in a region $A(A \subset I Z)$, we have [13]:

$$
P\left(N_{A}=k\right)=\frac{\left(\lambda_{i}\|A\|\right)^{k} \mathrm{e}^{-\lambda_{i}\|A\|}}{k !}
$$

The distribution of MSs in cell $j$ can! be described similarly. To collect subcarrier usage information in $\mathcal{I}_{j}$, each MS $\ell \in \mathcal{I}_{i}$ senses a an circular area of radius $r_{\ell}$ (denoted by the small circles shown in Fig. 1) and collects information on the existence and subcarrier usage of any MSs from $\mathcal{I}_{j}$ located within the circular sensing area.

Since the distribution of MSs and subcarrier usage in cell $j$ is geographically uniform, the completeness of the information collected on the subcarrier usage in $\mathcal{I}_{j}$ depends on the fraction of total area in the IZ jointly covered by MSs in $\mathcal{I}_{i}$. Let $p_{\mu} \in[0,1]$ describe such completeness, i.e., when $p_{\mu}=1$, all subcarrier usage information in $\mathcal{I}_{j}$ is completely collected. We can have

$$
\mathbb{E}\left[p_{\mu}\right]=\Upsilon_{A}=\frac{\left\|(\overbrace{\overbrace{1} \cup A_{2} \cdots \cup A_{\ell} \cdots}^{\text {all } \ell \in \mathcal{I}_{i}}) \cap A_{I Z}\right\|}{\left\|A_{I Z}\right\|},
$$

in which $\Upsilon_{A}$ is the fraction of area in the IZ covered by cognitive sensing; the expectation $\mathbb{E}[\cdot]$ is taken over the uniform distribution of subcarrier usage in $\mathcal{I}_{j} ; A_{\ell}$ is the sensing region covered by MS $\ell \in \mathcal{I}_{i}$ and $A_{I Z}$ denotes the region of IZ. A. Impact of Sensing Radius

In the general Boolean sensing model [13] where the distribution of sensors is governed by a Poisson point process, when sensors with sensing range $r$ are deployed over a large plane with density $\lambda$ (i.e., there are on average $\lambda$ sensors per unit area), a point is said to be "covered" if it falls within the sensing range of any sensor. As the plane enlarges $(\|A\| \rightarrow \infty)$, the probability that an arbitrary point on the plane is not "covered" can be computed from (1) as [13]:

$$
\begin{aligned}
P_{U C} & =\lim _{\|A\| \rightarrow \infty} \mathbb{E}\left[\left(1-\frac{\pi r^{2}}{\|A\|}\right)^{N_{A}}\right] \\
& \simeq \exp \left\{-\frac{\pi r^{2}}{\|A\|} \mathbb{E}\left[N_{A}\right]\right\} \\
& =\exp \left\{-\frac{\pi r^{2}}{\|A\|}(\lambda\|A\|)\right\}=\mathrm{e}^{-\lambda \pi r^{2}} .
\end{aligned}
$$

In our model, although MSs in $\mathcal{I}_{i}$ are distributed over (finite) IZ instead of infinite plane, (2) can still be used as a suitable approximation (its validity is verified later in this paper). To study the interaction between a BS and its MSs, we assume that all MSs in $\mathcal{I}_{i}$ choose the same sensing radius, i.e., $r_{\ell}=r, \forall \ell \in \mathcal{I}_{i}$. Such assumption allows us to investigate the behaviors of MSs as an integrated whole and model the multiple games played by a BS and different MSs as a single two player game (BS v.s. MSs). In examining the impact of the sensing radius $r$ chosen by a MS in $\mathcal{I}_{i}$, we define here the critical radius $\hat{r}$, such that

$$
\left|\mathcal{I}_{i}\right| \cdot \pi \hat{r}^{2}=\left\|A_{I Z}\right\|=f(\alpha) \pi R^{2},
$$

in which $f(\alpha)=\frac{2}{\pi} \cdot\left[\arccos \left(1-\frac{\alpha}{2}\right)-\left(1-\frac{\alpha}{2}\right) \sqrt{\left(4 \alpha-\alpha^{2}\right)}\right]$ is the fraction of the IZ area w.r.t. whole cell area. Therefore,

$$
\hat{r}=R \sqrt{\frac{f(\alpha)}{\left|\mathcal{I}_{i}\right|}}=\frac{R}{\sqrt{N_{i}}} .
$$


The critical radius $\hat{r}$ provides a theoretical lower bound on what radius should be chosen to cover the whole IZ without overlapping. However, we should note that given the relative irregularity between the circular shape of sensing region and the shape of IZ, it is impossible to cover IZ with exactly $\left|\mathcal{I}_{i}\right|$ circles of radius $\hat{r}$. However, $\hat{r}$ still serves a meaningful comparison base-line and can mask other system parameters from our analysis. Specifically, we can write the actual sensing radius as $r=\epsilon \hat{r}$, where $\epsilon \in \mathfrak{R}^{+}$is called the critical ratio. When $\epsilon \in[0,1]$, it describes the relative "inadequacy" of actual sensing radius, since the ideal lower bound is not yet met. On the other hand, when $\epsilon \in(1,+\infty)$, it describes a MS's willingness to intentionally provide relative "redundancy" in its sensing range, thereby increases the fraction of area in the IZ covered by its cognitive sensing. This way, more complete information on the subcarrier usage in $\mathcal{I}_{j}$ can be collected to facilitate more effective interference management at BS $i$.

With the definition of $\epsilon$ and $\hat{r}$, we can approximate $1-\mathbb{E}\left[p_{\mu}\right]$, the expected probability that an active subcarrier usage in $\mathcal{I}_{j}$ is not detected (i.e., the probability that the presence of a MS $\jmath \in \mathcal{I}_{j}$ in Fig. 1 is not detected) from (3):

$$
\begin{aligned}
1-\mathbb{E}\left[p_{\mu}\right] & \simeq P_{U C}=\mathrm{e}^{-\lambda_{i} \pi r^{2}} \\
& =\exp \left\{-\frac{N_{i}}{\pi R^{2}} \cdot \pi\left(\frac{R}{\sqrt{N_{i}}} \epsilon\right)^{2}\right\}=\mathrm{e}^{-\epsilon^{2}} .
\end{aligned}
$$

The accuracy of such approximation is evaluated in Fig. 2, in which the actual fraction of uncovered subcarrier usage is compared with the theoretical approximation in (6), when $N_{i}=64$ and $N_{i}=256$. We can see that the probability that any arbitrary subcarrier usage is not "covered" approaches 0 fast at an "exponentially-squared" rate. The simulation result matches the theoretical approximation quite well. In addition, we notice that as $N_{i}$ increases, theoretical approximation better matches simulation results. This roots from the fact that (3) is derived in infinite plane, which is not exactly true in our model. However, when $\epsilon$ is fixed, as $N_{i}$ increase and $\hat{r}$ decreases, IZ can be viewed as an "infinite" plane since $\lim _{N_{i} \rightarrow \infty} \hat{r} \rightarrow 0$. In this case, the sensing area covered by MSs but outside $A_{I Z}$ (and is thus ignored in our model) approaches zero, i.e., $\left(A_{1} \cup A_{2} \cdots \cup A_{\ell} \cdots\right)^{C} \cap A_{I Z} \rightarrow \emptyset$.

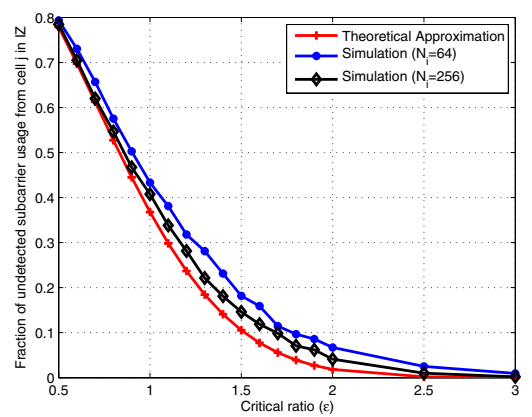

Fig. 2: Fraction of undetected subcarrier usage in $\mathcal{I}_{i}$, i.e., $1-\mathbb{E}\left[p_{u}\right]$, as a function of the critical ratio $\epsilon$.

\section{Stackelberg Game Between BS AND MSS}

\section{A. Reward from BS to Cognitive MS}

The task of cognitive sensing can be power-consuming for battery-operated MSs. Thus, to enable collection of subcarrier usage in $\mathcal{I}_{j}$, we assume that the BS will provide a power reward $^{3} \mathrm{P}_{\mathrm{R}}(r)=\mathrm{R}_{\mathrm{r}} r^{\rho}$ to a MS who performs cognitive sensing within a radius of $r . \mathrm{R}_{\mathrm{r}} \in \mathfrak{R}^{+}$is termed here the power reward factor, which describes how "generous" BS is in rewarding MS. $\rho$ is the pathloss exponent that usually takes value from $2 \sim 5$ and describes how power-consuming it is for an MS to perform cognitive sensing. Apparently, larger power rewards should be given when sensing is performed under adverse lossy wireless environment (e.g., $\rho=5$ ) than in $\operatorname{LoS}$ environments (e.g., $\rho=2$ ). Let $\mathrm{P}_{0}$ be the downlink transmission power allocated to a MS without cognitive sensing, the reward mechanism described above means that the BS will allocate power $\mathrm{P}_{0}+\mathrm{R}_{\mathrm{r}} r^{\rho}$ to a MS that performs cognitive sensing within a radius of $r$.

\section{B. Tradeoffs for BS and $M S$}

The reason that BS promises power rewards to MSs that perform cognitive sensing is that information collected through the sensing can be used to reduce the power wasted in downlink transmission due to subcarrier collisions. Here we assume when the same subcarrier is allocated to different MSs co-located in the IZ (BS $i$ to a MS $\ell \in \mathcal{I}_{i}$ and BS $j$ to another MS $\jmath \in \mathcal{I}_{j}$ ), the collision results in total waste in transmission power on this subcarrier. In addition, when perfectly accurate information on the subcarrier usage in $\mathcal{I}_{j}$ can be obtained, we assume all collisions can be avoided ${ }^{4}$. That is, subcarrier collision occurs only due to incompleteness in the knowledge of subcarrier usage in $\mathcal{I}_{j}$. In this case, the net power saving for the BS (from rewarding cognitive sensing) can be given as

$$
\mathrm{P}_{\mathrm{BS}}^{\mathrm{s}}=\beta_{j} f(\alpha) \mathrm{P}_{0}-\beta_{j} f(\alpha) \mathrm{e}^{-\epsilon^{2}}\left[\mathrm{P}_{0}+\mathrm{R}_{\mathrm{r}}(\epsilon \hat{r})^{\rho}\right] .
$$

For the MS, the drawback of sensing is the power consumption associated with it, which increases with $r$ and can be modeled as $\mathrm{P}_{S}(r)=\mathrm{C}_{1} r^{\rho}$, where $\mathrm{C}_{1}$ is the power consumed in sensing within a unit-radius circular area. On the other hand, benefits of sensing are two-folded:

- Firstly, the power reward by BS is beneficial to the MS and can (at least partially) compensate for the batterypower consumed by MS to perform sensing.

- Secondly, the information collected can be compiled at BS and used to reduce subcarrier collision for MS.

To model the benefit from BS's reward, we let $\delta_{e} \in \mathfrak{R}^{+}$be the "exchange rate" from the saving in battery power to the increase in downlink transmission power. That is, MS views $\mathrm{P}_{R}$ from BS equivalently as saving in its own battery power, in the amount of $\frac{\mathrm{R}_{\mathrm{r}} r^{\rho}}{\delta_{e}}$. In other words, a rational MS is willing to consume $\frac{\mathrm{R}_{\mathrm{r}} r^{\rho}}{\delta_{e}}$ units of power in cognitive sensing in exchange for power reward of $\mathrm{R}_{\mathrm{r}} r^{\rho}$ from the BS. When $\delta_{e}>1$, the MS values its own battery power over the increment in downlink power allocated to it, and vice versa. On the other hand, a MS also disfavors wasting its power in receiving undecodable (due to collision) downlink transmission. In this regard, let $\mathrm{C}_{2}^{\prime}$ be the power wasted in receiving a collided (thus undecodable)

\footnotetext{
${ }^{3}$ Note that different functional forms of $\mathrm{P}_{\mathrm{R}}(r)$ can be chosen other than the one provided here, with the general principal that $\frac{\partial \mathrm{P}_{R}(r)}{\partial r}>0$.

${ }^{4}$ Such assumption is valid when BS $i$ has enough flexibility in avoiding subcarrier collision if perfect information is available. It is taken here to facilitate investigating the impact of "completeness" in information collecting.
} 
subcarrier and $\mathrm{C}_{2}=\mathrm{C}_{2}^{\prime} \beta_{j} f(\alpha)$. Then the expected amount of wasted power can be given as $\mathrm{P}_{W}=\mathrm{C}_{2} \mathrm{e}^{-\epsilon^{2}}$.

With all the tradeoffs, the equivalent net power saving for an MS in performing cognitive sensing within radius $r$ is:

$$
\mathrm{P}_{\mathrm{MS}}^{\mathrm{s}}=\mathrm{C}_{2}\left(1-\mathrm{e}^{-\epsilon^{2}}\right)+\left(\frac{\mathrm{R}_{\mathrm{r}}}{\delta_{e}}-\mathrm{C}_{1}\right) \cdot(\epsilon \hat{r})^{\rho} .
$$

\section{Strategy Spaces and Utility Functions}

Different system parameters are involved in (7) and (8) to describe the payoffs of BS and MS. To model the interactions between a BS and a MS as a strategic game, the strategy spaces for the two players (i.e., what parameters are at the liberty of each player and what are not) should be first identified. In the system described above, $\mathrm{C}_{1}$ is the power required to sense a circular region of unit radius, $\mathrm{C}_{2}$ is the the amount of power wasted in receiving an undecodable (i.e., collided) subcarrier. Both of them are determined by the hardware design of the $\mathrm{MS}$, and are not subject to variations after deployment. Clearly, $\mathrm{C}_{2}=\beta_{j} f(\alpha) \mathrm{C}_{2}^{\prime}$ cannot be altered by either MS or BS in cell $i$. Also, as can be seen from (5), $\hat{r}$ is determined by system deployment and the pathloss exponent $\rho$ is by definition a subjective description of the MS's wireless environment. These parameters, therefore, cannot serve as decision variables. On the other hand, although the parameters $\mathrm{P}_{0}$ and $\delta_{e}$ can each be used to partially describe the preference of one player, neither of them affects the payoffs of both players. Thus, they do not capture the essential interactive nature of the decision making process and are excluded here from the strategy spaces.

After close scrutiny of different parameters, the strategy spaces of BS and MS can be given as $\left\{R_{\mathrm{r}}: \mathrm{R}_{\mathrm{r}} \in \mathfrak{R}^{+}\right\}$ and $\left\{\epsilon: \epsilon \in \mathfrak{R}^{+}\right\}$, respectively. That is, BS decides on the power reward factor $R_{\mathrm{r}}$ while MS decides on the critical ratio $\epsilon$ (which eventually determines actual sensing radius). Each of these two decision parameters affects payoffs of both BS and MS. If we assume hereafter that $\rho=2$, the utility functions (which each rational player seeks to maximize) for the two players can be given, from simplification of (7) and (8), as

$$
\mathrm{U}_{\mathrm{BS}}\left(\epsilon, \mathrm{R}_{\mathrm{r}}\right)=\mathrm{P}_{0}-\mathrm{e}^{-\epsilon^{2}} \cdot\left[\mathrm{P}_{0}+\mathrm{R}_{\mathrm{r}}(\epsilon \hat{r})^{2}\right]
$$

and

$$
\mathrm{U}_{\mathrm{MS}}\left(\epsilon, \mathrm{R}_{\mathrm{r}}\right)=\left(\frac{\mathrm{R}_{\mathrm{r}}}{\delta_{e}}-\mathrm{C}_{1}\right)(\epsilon \hat{r})^{2}-\mathrm{C}_{2} \mathrm{e}^{-\epsilon^{2}} .
$$

\section{Stackelberg Game between BS and MS}

We now model the interactive decision making between BS and MS as a Stackelberg leader-follower game. Specifically, we assume that BS is the "leader" that makes decision first, by determining the value of $R_{r}$. MS is the "follower" that chooses its optimal strategy on $\epsilon$ upon observation of $\mathrm{R}_{\mathrm{r}}$, thus determines the sensing radius $r=\epsilon \hat{r}$.

The algorithm for this Stackelberg game that obtains Nash Equilibrium is given as Algorithm III.1. The game begins with the decision from BS. However, since the utility of BS is determined jointly by $\epsilon$ and $R_{r}$, rational decision making is only possible with knowledge on what it will trigger from the MS on $\epsilon$. To this end, BS assumes rationality in MS and predicts that MS will choose the response that maximizes MS's own utility. That is, BS assumes the "rational response" of MS, which varies with $R_{\mathrm{r}}$, will be $\widetilde{\epsilon}\left(\mathrm{R}_{\mathrm{r}}\right)$, as shown by the statement (i) in Algorithm III.1. BS then substitutes $\widetilde{\epsilon}\left(\mathrm{R}_{\mathrm{r}}\right)$ into (10) and transforms its utility function $\mathrm{U}_{\mathrm{BS}}\left(\epsilon, \mathrm{R}_{\mathrm{r}}\right)$ into a single variable function $\mathrm{U}_{\mathrm{BS}}^{*}\left(\mathrm{R}_{\mathrm{r}}\right)$ (statement (ii)). Finally $\mathrm{BS}$ can determine its own optimal strategy $\mathrm{R}_{r}^{*} \in \mathfrak{R}^{+}$that maximizes $\mathrm{U}_{\mathrm{BS}}^{*}\left(\mathrm{R}_{\mathrm{r}}\right)$ (statement (iii)).

On the other hand, after MS observes the decision $\mathrm{R}_{\mathrm{r}}^{*}$ from $\mathrm{BS}$, it can directly choose its optimal strategy (response) $\epsilon^{*}$ which maximizes its own utility (statement (iv)). For rational $\mathrm{BS}$ and MS, when all other system parameters $\left(\mathrm{C}_{1}, \mathrm{C}_{2}, \delta_{e}\right.$, etc.) are available to both players, we would have $\epsilon^{*}=\widetilde{\epsilon}\left(\mathrm{R}_{\mathrm{r}}^{*}\right)$. From this process of decision making, we see that the outcome $\left(\epsilon^{*}, \mathrm{R}_{\mathrm{r}}^{*}\right)$ is the unique Nash Equilibrium of this game by definition [11], since neither players can be better off by deviating alone from such outcome.

Proposition 3.1: For the Stackelberg game modeled above, the rational response function of a MS can be described as:

- If $\mathrm{R}_{r} \geq \delta_{e} \mathrm{C}_{1} \geq 0$, each MS always performs cognitive sensing with the maximal sensing radius, i.e., $\widetilde{\epsilon}\left(R_{r}\right) \rightarrow$ $+\infty, \forall \mathrm{R}_{\mathrm{r}} \in\left\{X \mid X \geq \delta_{e} \mathrm{C}_{1} \geq 0\right\}$, unless restrained by its sensing capacity in practice;

- If $0<\mathrm{C}_{2}<\left(\mathrm{C}_{1}-\frac{\mathrm{R}_{r}}{\delta_{e}}\right) \hat{r}^{2}$, MS never performs any cognitive sensing, i.e., $\epsilon=0, \forall \mathrm{R}_{\mathrm{r}} \in\{X \mid X<$ $\left.\delta_{e}\left(\mathrm{C}_{1}-\frac{\mathrm{C}_{2}}{\hat{r}^{2}}\right)\right\} \bigcap \mathfrak{R}^{+}$; and

- If $0<\left(\mathrm{C}_{1}-\frac{\mathrm{R}_{r}}{\delta_{e}}\right) \hat{r}^{2}<\mathrm{C}_{2}$, MS will perform cognitive sensing with a radius of $r=\widetilde{\epsilon}\left(\mathrm{R}_{\mathrm{r}}\right) \hat{r}$, in which

$$
\widetilde{\epsilon}\left(\mathrm{R}_{\mathrm{r}}\right)=\sqrt{\ln \frac{\mathrm{C}_{2}}{\left(\mathrm{C}_{1}-\frac{\mathrm{R}_{r}}{\delta_{e}}\right) \hat{r}^{2}}},
$$

Sketched Proof: We can show through straightforward derivation that $\mathrm{U}_{\mathrm{MS}}\left(\epsilon, \mathrm{R}_{\mathrm{r}}\right)$ will be monotonic (increasing and decreasing, respectively) in the first two cases w.r.t. $\epsilon$, therefore the boundary maximals. When $0<\left(\mathrm{C}_{1}-\frac{\mathrm{R}_{r}}{\delta_{e}}\right) \hat{r}^{2}<\mathrm{C}_{2}$,

$$
\widetilde{\epsilon}=\arg \max _{\epsilon \in \mathfrak{R}^{+}} \mathrm{U}_{\mathrm{MS}}\left(\epsilon, \mathrm{R}_{\mathrm{r}}\right)=\left\{\epsilon: \frac{\partial \mathrm{U}_{\mathrm{MS}}}{\partial \epsilon}=0\right\} .
$$

By solving $\frac{\partial\left\{\left(\mathrm{C}_{1}-\frac{\mathrm{R}_{r}}{\delta_{e}}\right)(\hat{r} \epsilon)^{2}\right\}}{\partial \epsilon}+\frac{\partial\left\{\mathrm{C}_{2} \mathrm{e}^{-\epsilon^{2}}\right\}}{\partial \epsilon}=0$, we have $\widetilde{\epsilon}_{1}=$ $\sqrt{\ln \frac{\mathrm{C}_{2}}{\left(\mathrm{C}_{1}-\frac{\mathrm{R} r}{\delta_{e}}\right) \hat{r}^{2}}}$ or $\widetilde{\epsilon}_{2}=0$. It can be easily verified that $\widetilde{\epsilon}_{2}=0$ is not a valid maximum of $\mathrm{U}_{\mathrm{MS}}$. Therefore $\widetilde{\epsilon}\left(\mathrm{R}_{\mathrm{r}}\right)=\widetilde{\epsilon}_{1}$ is the rational response function for MS can take in this case.

The first case in Proposition 3.1 corresponds to the ideal (and usually unrealistic) scenario in which the cost of sensing can always be fully (or even over) compensated by the reward from BS. The second case describes the scenario when sensing cost is always too high to be justified. These two extreme cases are trivial in our investigation and we will hereafter focus on the last case where different choices of $\epsilon$ affect the profitability of cognitive sensing. Substituting (11) into (10) yields

$$
\begin{aligned}
\mathrm{U}_{\mathrm{BS}}^{*}\left(\mathrm{R}_{\mathrm{r}}\right) & =\left.\mathrm{U}_{\mathrm{BS}}\left(\epsilon, \mathrm{R}_{\mathrm{r}}\right)\right|_{\epsilon=\widetilde{\epsilon}\left(\mathrm{R}_{\mathrm{r}}\right)} \\
& =\mathrm{P}_{0}-\frac{\left(\mathrm{C}_{1}-\frac{\mathrm{R}_{r}}{\delta_{e}}\right) \hat{r}^{2}}{\mathrm{C}_{2}}\left[\mathrm{P}_{0}+\hat{r}^{2} \mathrm{R}_{\mathrm{r}} \cdot \ln \frac{\mathrm{C}_{2}}{\left(\mathrm{C}_{1}-\frac{\mathrm{R}_{r}}{\delta_{e}}\right) \hat{r}^{2}}\right] .
\end{aligned}
$$

The maximization of $\mathrm{U}_{\mathrm{BS}}^{*}\left(\mathrm{R}_{\mathrm{r}}\right)$ can be obtained by solving $\frac{\partial \mathrm{U}_{\mathrm{BS}}^{*}}{\partial \mathrm{R}_{\mathrm{r}}}=0$, which is equivalent to solving

$$
\frac{f_{1}(\cdot) \hat{r}^{2}+\mathrm{P}_{0}}{\delta_{e}}=\left(\mathrm{C}_{1}-\frac{\mathrm{R}_{\mathrm{r}}}{\delta_{e}}\right) \cdot f_{2}(\cdot)
$$

for $\mathrm{R}_{\mathrm{r}}$, in which $f_{1}(\cdot)=\mathrm{R}_{\mathrm{r}} \cdot \ln \left[\mathrm{g}\left(\mathrm{R}_{\mathrm{r}}\right)\right], f_{2}(\cdot)=\frac{\partial f_{1}(\cdot)}{\partial \mathrm{R}_{\mathrm{r}}}=$ $\ln \left[\mathrm{g}\left(\mathrm{R}_{\mathrm{r}}\right)\right]-\frac{\mathrm{R}_{\mathrm{r}} \hat{r}^{2}}{\mathrm{~g}\left(\mathrm{R}_{\mathrm{r}}\right) \delta_{e}}$ and $\mathrm{g}\left(\mathrm{R}_{\mathrm{r}}\right)=\frac{\mathrm{C}_{2}}{\left(\mathrm{C}_{1}-\frac{\mathrm{R}_{r}}{\delta_{e}}\right) \hat{r}^{2}}$. The derivation 
Algorithm III.1: Stackelberg Leader-Follower Game Between BS AND MS $\left(\hat{r}, \mathrm{P}_{0}, \mathrm{C}_{1}, \mathrm{C}_{2}, \delta_{e}, \rho\right)$

comment: Implement Stackelberg leader-follower game with continuous strategy spaces.

initialization: The values of $\hat{r}, \mathrm{C}_{1}, \mathrm{C}_{2} \mathrm{P}_{0}$, and $\delta_{e}$ are determined.

for the leader (BS) :

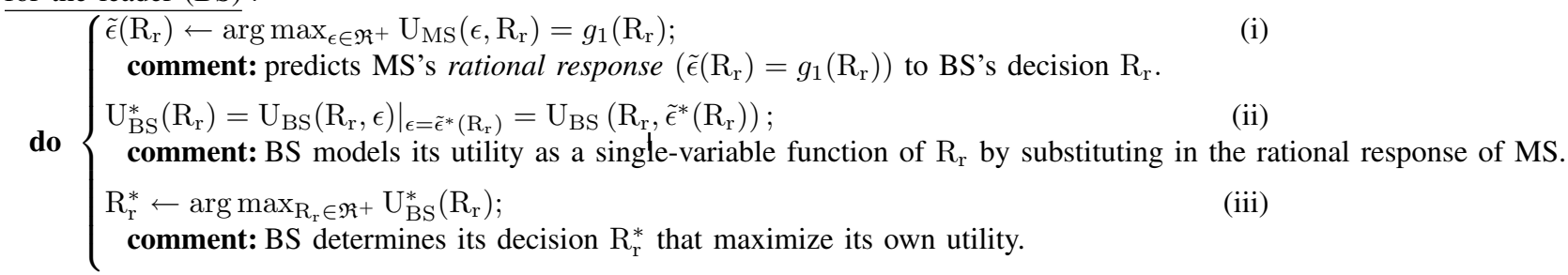

for the follower (MS) :

do $\epsilon^{*} \leftarrow \arg \max _{\epsilon \in \mathfrak{R}+} \mathrm{U}_{\mathrm{MS}}\left(\epsilon, \mathrm{R}_{\mathrm{r}}^{*}\right)$;

comment: MS makes decision upon observation of the decision $\mathrm{R}_{\mathrm{r}}^{*}$ from BS.

output (utility pair $\left\{\mathrm{U}_{\mathrm{BS}}\left(\epsilon^{*}, \mathrm{R}_{\mathrm{r}}^{*}\right), \mathrm{U}_{\mathrm{MS}}\left(\epsilon^{*}, \mathrm{R}_{\mathrm{r}}^{*}\right)\right\}$ ).

of a closed-form $\mathrm{R}_{r}^{*}$ from (14) is untractable with general parameter settings. In addition, local optimal and global optimal should be carefully handled. Yet such evaluation can be numerically implemented in practice. Especially when it is performed at BS where computational capacity is abundant.

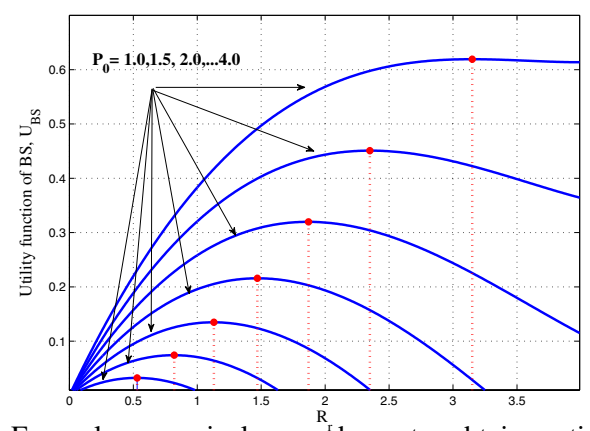

Fig. 3: Example numerical procédures to obtain optimal $R_{r}^{*}$ by evaluating equation (13) under different $\mathrm{P}_{0}$.

We show in Fig. 3 an example to obtain $R_{r}^{*}$ by numerical evaluation, under the condition that $0<\left(\mathrm{C}_{1}-\right.$ $\left.\frac{\mathrm{R}_{r}}{\delta_{e}}\right) \hat{r}^{2}<\mathrm{C}_{2}$. Specifically, we choose the parameters such that $\mathrm{C}_{1}=\mathrm{C}_{2}=8, \hat{r}=\delta_{e}=1$ and $\mathrm{P}_{0}$ varies from 1.0 to 4.0 . Fig. 3 shows the function $\mathrm{U}_{\mathrm{BS}}^{*}\left(\mathrm{R}_{\mathrm{r}}\right)$ assuming rational response from $\mathrm{MS}$, which is derived in (13). The optimal strategy of BS can then be determined from Fig. 3 as $\mathrm{R}_{\mathrm{r}}^{*}=[0.53,0.82,1.13,1.47,1.87,2.35,3.15]$ when $\mathrm{P}_{0}$ varies from 1.0 to 4.0 in increment of 0.5 . This suggests that in this specific system setting, the optimal reward factor $\mathrm{R}_{\mathrm{r}}^{*}$ increases monotonically with $\mathrm{P}_{0}$. It correlates positively with the intuition since as the base transmission power $\mathrm{P}_{0}$ increases, the cost of collision increases. In this case, BS will be willing to provide more "generous" reward to encourage cognitive sensing by MS, which eventually facilitates reducing subcarrier collisions. The relationship between the optimal decision and other system parameters can be evaluated similarly by BS.

In contrast, the decision making process at MS is much less computationally complex. In fact, upon observing the decision (reward factor) from BS (i.e., $R_{r}=R_{r}^{*}$ ), MS can make its decision directly from the guidelines laid out in
Proposition 3.1. The imbalance in computational complexity in the decision making process conforms with the general design principles that allow computational complexity at the BS and reduce it at the MS.

\section{CONCLUSIONS}

We formulate a game-theoretic framework using Stackelberg game between BS and MS, to facilitate inter-cell interference management through the means of cognitive sensing performed by MSs. Tradeoffs for the players (BS and MS) in this game are investigated, which are used to properly model their utility functions. The algorithm to implement such game to achieve Nash Equilibrium is given and behaviors of players in this game are derived and discussed.

\section{REFERENCES}

[1] "IEEE Standard for Local and Metropolitan Area Networks - Part 16: Air Interface for Fixed Broadband Wireless Access Systems," October 2004;

[2] "IEEE Standard for Local and Metropolitan Area Networks - Part 16: Air Interface for Fixed Broadband Wireless Access Systems, Amendment 2: Physical and Medium Access Control Layers for Combined Fixed and Mobile Operation in Licensed Bands," February 2006;

[3] 3GPP technical Report, TR 25.913 version 2.1.0 " Requirements for Evolved UTRA and UTRAN ", 3GPP TSG RAN 28, Quebec, Canada, June 1-3, 2005, Tdoc RP-050384;

[4] 3GPP, TR 25.814, "Physical Layer Aspects for Evolved UTRA," V7.0.0;

[5] Huawei, "3GPP R1- 050507: Soft Frequency Reuse Scheme for UTRAN LTE,” TSG RAN WG1 Meeting 41, Athens, Greece, May. 2005;

[6] Siemens, "3GPP: R1-060135: Interference Mitigation by Partial Frequency Reuse," TSG-RAN WG1 Ad Hoc Meeting on LTE, Helsinki, Finland, January 2006;

[7] M. Sternad, T. Ottosson, A. Ahlen, A. Svensson, "Attaining both Coverage and High Spectral Efficiency with Adaptive OFDM Downlinks," IEEE Vehicular Technology Conference, Orlando, Oct. 2003;

[8] J.H Yeom, Y.H. Lee, "Mitigation of inter-cell interference in the WiMAX system," IEEE Mobile WiMAX Symposium, March 2007 Page(s):26 - 31;

[9] Y. Chen, K. H. Teo, S. Kishore, and J. Zhang, "Inter-Cell Interference Management in WiMAX Downlink by Inter-BSs Stackelberg Game," ICC 2008, accepted;

[10] I. Katzela and M. Naghshineh, "Channel Assignment Schemes for Cellular Mobile Telecommunication Systems: a Comprehensive Survey," IEEE Personal Communications, vol. 3, no. 3, pp. 10-31, June 1996;

[11] Martin J. Osborne, An introduction to game theory, Oxford University Press, August 2003;

[12] S. Haykin, "Cognitive radio: brain-empowered wireless communications," Selected Areas in Communications, IEEE Journal on, Volume 23, Issue 2, Feb. 2005 Page(s): 201 - 220;

[13] P. Hall, Intro. to the Theo. of Coverage Proc., John Wiley \& Sons, 1998. 